\title{
Automated Preparation of Nerve Tissue for TEM
}

Thomas Strader ${ }^{1}$ and Benjamin August ${ }^{2}$

${ }^{1}$ Microscopy Innovations, LLC, Marshfield, Wisconsin, United States, ${ }^{2}$ University of WisconsinMadison, Madison, Wisconsin, United States

Nerve tissue is imaged using transmission electron microscopy (TEM) for myriad reasons including diagnosis, research and education. A new system developed by scientists at the UW-Madison and Microscopy Innovations (Marshfield, WI) can process from 1 to 24 nerve specimens, from fix-rinse through infiltration, in 37 minutes fully unattended, with excellent results.

A critical challenge when preparing rat tibial nerve specimens for TEM is their small size and lack of rigidity. Researchers at the University of Wisconsin-Madison and Microscopy Innovations have developed a method to process these unique specimens for rapid, walk-away sample processing using the Microscopy Innovations specimen (S-) capsule and the ASP-1000 Automated Specimen Processor. The S-capsule securely holds each delicate specimen for the liquid handling steps and keeps them separated and sequestered for the duration of the liquid handling steps performed on the sample handling robot.

In a batch of 8, perfusion-fixed rat tibial nerve specimens were collected and stored in buffer. Immediately prior to processing, the specimens were removed from buffer, trimmed and placed in Specimen Capsules (p/n 22200) from Microscopy Innovations for safe and secure processing. Specimen length and width length varied but was approximately $2 \mathrm{~mm}$ by $3 \mathrm{~mm}$ in most cases.

Bar-code labelled S-capsules loaded with the tibial nerve specimens were then mounted on the ASP-1000 automated specimen processing robot. The robot deck was pre-loaded with reagent reservoirs containing all of the reagents and rinsing solutions needed to prepare the specimens for imaging by TEM. The integrated enclosure and effective fume removal system kept the system operators and other lab inhabitants safely protected from the hazardous fumes. The pre-programmed protocol was initiated by the system operator. The specimens were then subjected to: rinse of the fixative/buffer, osmification, en-bloc uranyl acetate staining, dehydration and infiltration.

Following robotic processing, the capsules were polymerized overnight at $60^{\circ} \mathrm{C}$. The next morning the resin-filled s-capsules (i.e. "blocks" at this point) were trimmed, and sectioned. No post-staining was performed. 

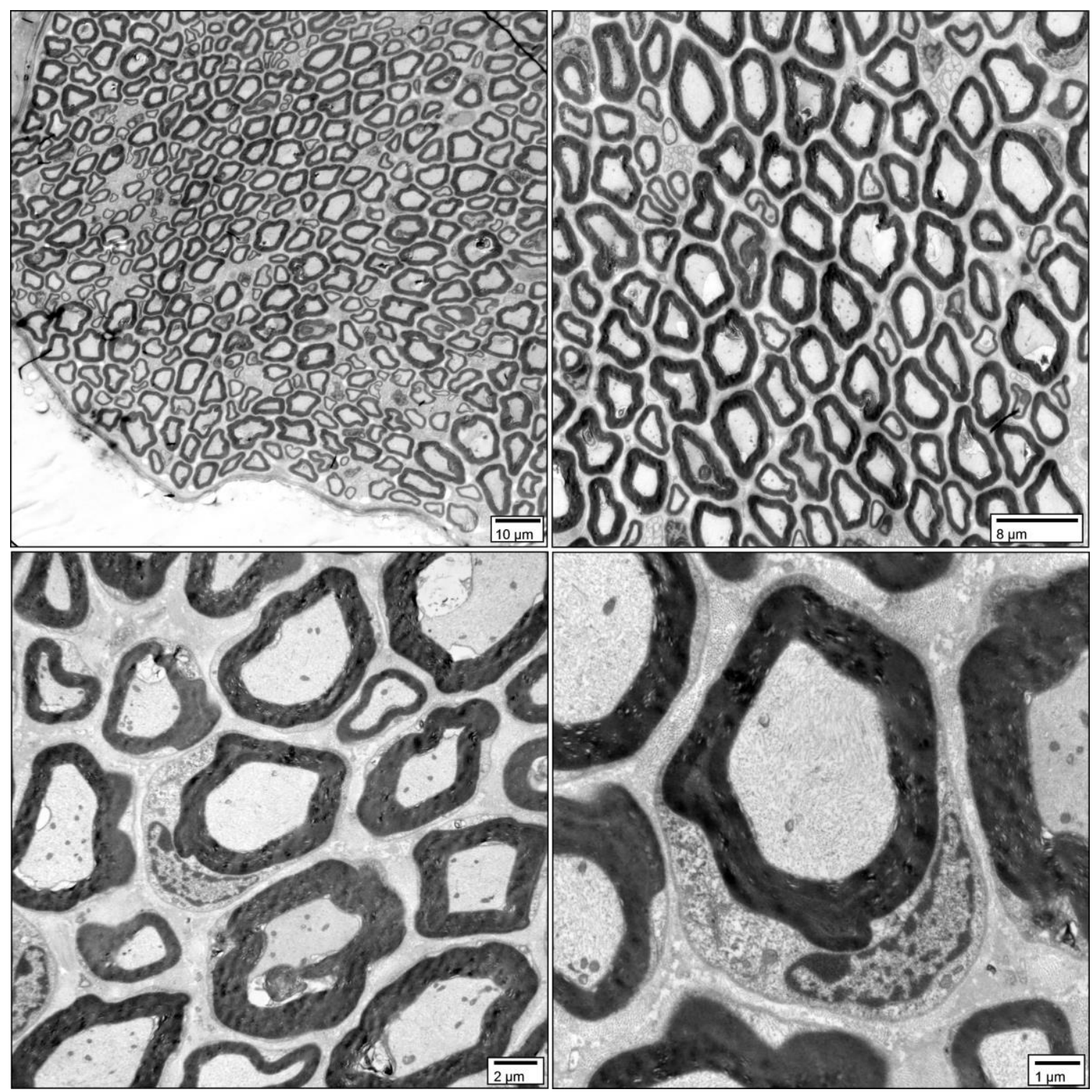

Figure 1. Four images mages collected in a Phillips 120 STEM at four different magnifications ( 1um, 2 um, $8 \mathrm{um}$ and $10 \mathrm{um}$ reference bars shown) of tibial nerve bundle. In the bottom right panel, note the white axon in the middle surrounded by the well-defined dark myelin sheath, with Schwann cell enveloping the lower half of the cross-section. 